\title{
An image analysis method to quantify the lateral pivot shift test
}

\author{
Yuichi Hoshino $\cdot$ Paulo Araujo $\cdot$ James J. Irrgang $\cdot$ \\ Freddie H. Fu $\cdot$ Volker Musahl
}

Received: 10 July 2011/Accepted: 15 December 2011/Published online: 28 December 2011

(C) The Author(s) 2011. This article is published with open access at Springerlink.com

\begin{abstract}
Purpose Although various kinematic measurements with advanced technology have been used for quantitative evaluation of the pivot shift test, there is no clinically available quantification method of the pivot shift test. The purpose was to describe a novel image analysis technique for quantitative assessment of the pivot shift test using universally available and affordable devices.

Methods Five ACL deficient knees were tested during examination under anesthesia. Three skin markers were attached to bony landmarks on the lateral side of the knee joint, (1) Gerdy's tubercle, (2) fibular head, and (3) lateral epicondyle. A standard digital video camera captured motion of the lateral aspect of the knee during the pivot shift test. The image was processed into a 2-dimensional (2-D) coordinate system with Image $\mathbf{J}$ software (National Institute of Health, USA) to trace the three landmarks. The anteroposterior (AP) position of the femur was calculated on consecutive still images extracted from the video recording. AP translation over time was reported.

Results The reduction phase of the pivot shift could be tracked consistently by a sudden anterior translation of the distal femur. The sudden anterior translation of the lateral epicondyle was on average $3.7 \pm 2.1 \mathrm{~mm}$ and occurred within $0.2 \pm 0.1 \mathrm{~s}$ from the start of this anterior translation till the end.
\end{abstract}

Y. Hoshino · P. Araujo · J. J. Irrgang · F. H. Fu · V. Musahl Department of Orthopaedic Surgery, University of Pittsburgh, Pittsburgh, PA, USA

F. H. Fu ( $\square)$

Kaufmann Medical Building, Suite 1011, 3471 Fifth Avenue, Pittsburgh, PA 15213, USA

e-mail: ffu@msx.upmc.edu; ffu@upmc.edu
Conclusion The sudden shift of the lateral compartment of the knee joint was successfully detected by this newly developed image analysis measurement method. This image analysis technique facilitates a simple and affordable method to evaluate the lateral pivot shift test.

Level of evidence Diagnostic studies, Level IV.

Keywords Pivot shift test - Quantitative measurement . Image analysis $\cdot$ ACL

\section{Introduction}

The lateral pivot shift test $[5,6]$ is widely used to evaluate rotational instability after ACL injury and treatment. The result of this test is subjectively determined by the examiner and therefore highly variable [13]. There is no quantitative evaluation system that can be used in clinical practice despite extensive research efforts to measure this instability.

The primary reason for the difficulty to establish an evaluation system is the complexity of the pivot shift movement that is composed of a six degree-of-freedom tibial internal-external (i-e) rotation, varus-valgus $(v-v)$ rotation, and anterior-posterior $(\mathrm{a}-\mathrm{p})$ translation [3]. Bedi et al. [1] used a navigation system to demonstrate a good correlation between $a-p$ translation of the lateral compartment and the results of the clinical pivot shift test grade. The results of this study indicated that each clinical graded correlated to an increment of approximately $6 \mathrm{~mm}$ of lateral compartment translation [1]. This suggests that the lateral compartment translation could reflect the clinical pivot shift test grade.

Most reported devices used in experimental trials of pivot shift measurement, such as navigation system [1, 2, 9, 16], electromagnetic sensor [4, 7], and accelerometer [10, 
11], are not universally available and associated with considerable costs. Digital camera technology was developed over the past 20 years and today is available with high-resolution digital images $(10.0$ Mpixel) for $<100$ dollars. Digital video recordings can be processed with free-of-charge image analysis software.

This report describes a newly developed image analysis for capturing the lateral pivot shift movement using simple and affordable devices.

\section{Materials and methods}

Capture of the video image during the pivot shift test

Ring-shaped white stickers $14 \mathrm{~mm}$ in diameter (white portable reinforcements, item \#636156, Staples, Inc.,

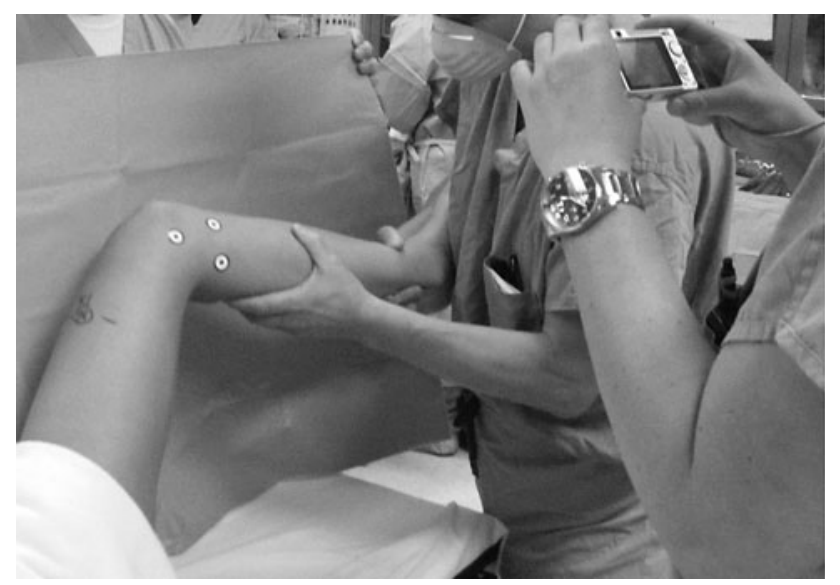

Fig. 1 Manual pivot shift test is captured with standard digital camera. Three points are tracked, (1) lateral epicondyle, (2) Gerdy tubercle, and (3) fibula head
Framingham, MA, USA) were attached as skin markers on the bony landmarks, (1) lateral epicondyle, (2) Gerdy's tubercle, and (3) fibular head. The distance between the centers of the markers located over Gerdy's tubercle and the fibular head was preliminarily measured by a ruler.

A video recording of the lateral aspect of the knee during the manually performed pivot shift test is taken by an ordinary digital camera (Cyber-shot ${ }^{\circledR}$ W120 Digital, Sony, Tokyo, Japan). The pivot shift test is performed with the maneuver reported by Galway and McIntosh [5] (Fig. 1). The camera angle and distance to the knee is determined as to capture the three skin markers as clearly as possible in the center of the frame throughout the pivot shift test. The background behind the knee is covered by a monotone sheet to reduce noise during the processing stage.

Image analysis using NIH image $\mathbf{J}$ software (National Institute of Health, Bethesda, MD, USA)

The video recording containing the pivot shift test is reformatted as avi file, which is compatible with Image J. This movie file is trimmed to have one sequence of the pivot shift test, which normally takes about $1 \mathrm{~s}$ to perform.

The avi file is opened as a stack of images by Image $\mathbf{J}$ (Fig. 2a). The images are cropped to capture only the three markers within the imaging frames throughout the test (Fig. 2b). The color information of the images is converted to 8-bit grayscale, and the brightness and contrast are adjusted to highlight the skin markers. The areas of the skin markers are selected by the area detection tool by the level of grayscale. The detecting threshold of the grayscale level is adjusted to maximize the areas of the markers and to minimize the unwanted area selection (Fig. 2c). The $X Y$

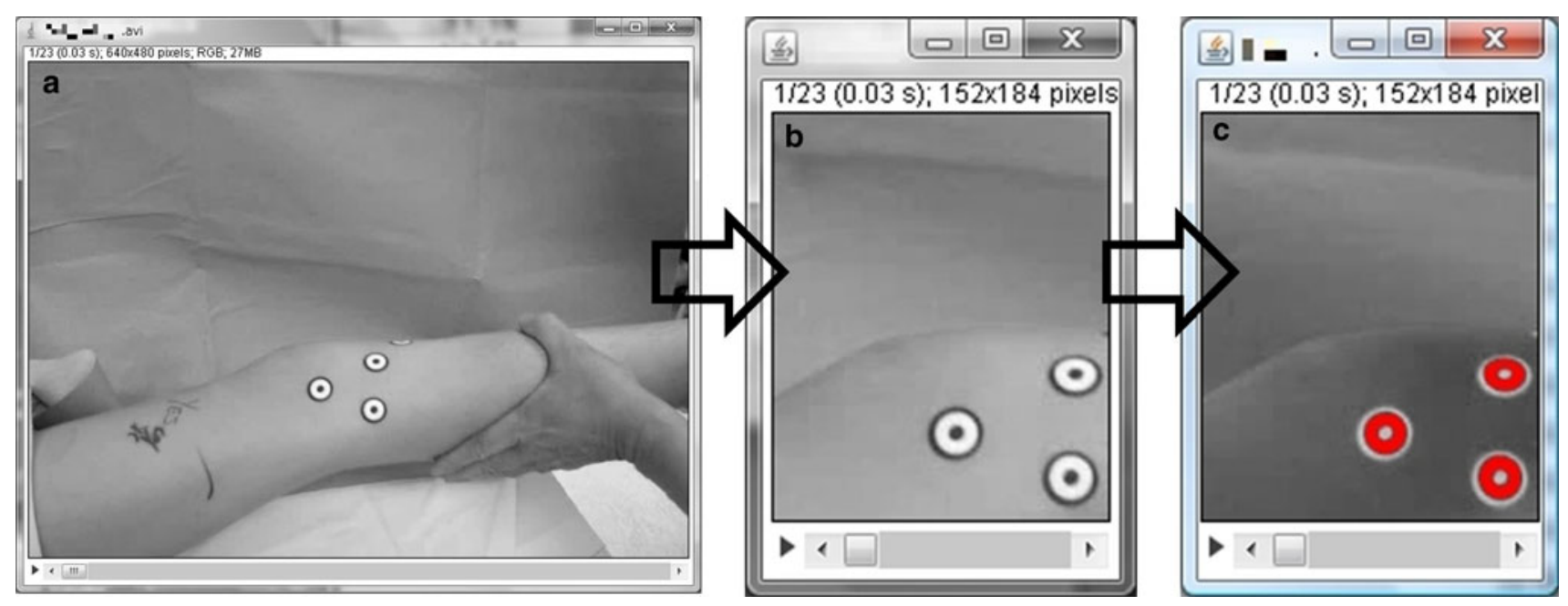

Fig. 2 a A picture processed from a movie of the pivot shift test with image processing software (ImageJ). b A cropped picture with clearly captured skin markers. $\mathbf{c}$ The areas of the skin markers are detected and colored as red 
plots of the centroid of the detected areas and the outlines of the detected area are provided after conducting particle analysis. The $X Y$ plots of the centroids are used to calculate the $a-p$ translation of the lateral compartment. The outline data facilitate identification of each detected skin marker area and the corresponding $X Y$ plot of its centroid location (Fig. 3).

\section{Calculation of lateral compartment translation}

In each frame, the $X Y$ plot of the intersection point, termed as "pivot point $(\mathrm{P})$ " between the tibial horizontal line,

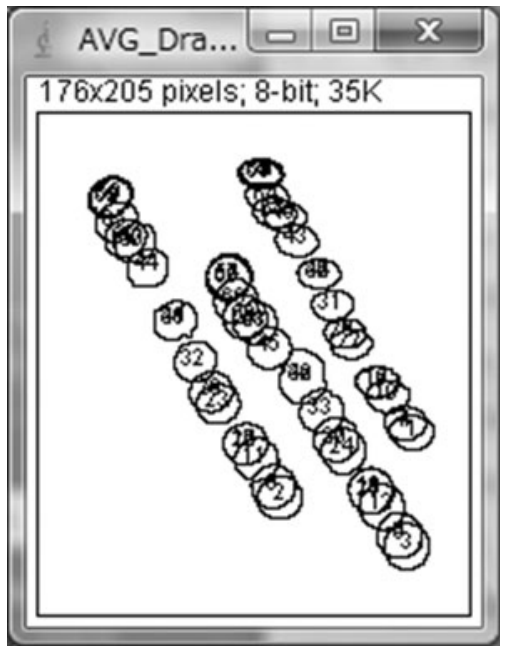

Fig. 3 Outline drawings of the detected areas in consecutive frames during the test (in this case, $n=13$ frames were processed during the pivot shift)

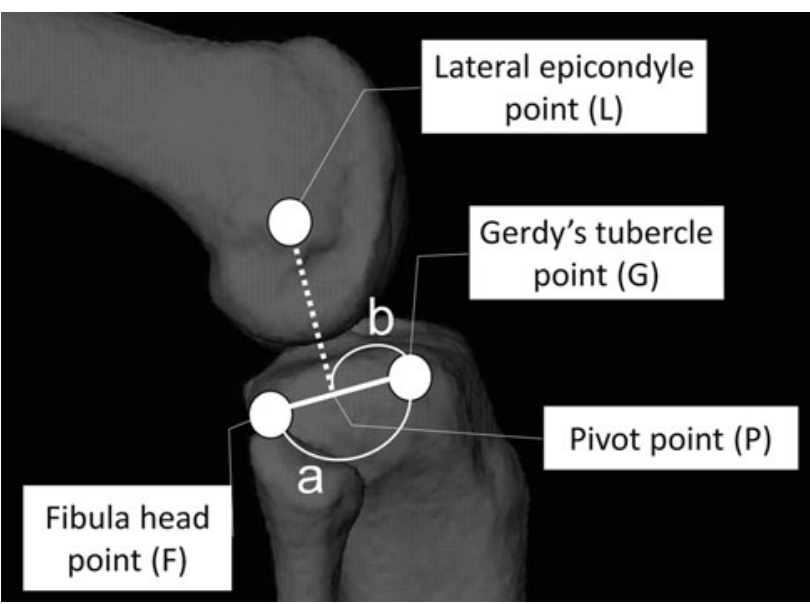

Fig. 4 Calculation of the position of the distal femur. Using ImageJ software, a line is drawn to connect Gerdy's tubercle (G) with the fibula head (F; line a). A perpendicular line is drawn from the lateral epicondyle (L) to line $a$. The intersection between the two lines is termed "pivot point" (P). A third line is drawn from G to $\mathrm{P}$ (line $b$ ). The position of the distal femur is calculated as line [ $(a$ actual measurement $) \times(b / a$ from image analysis $)]$

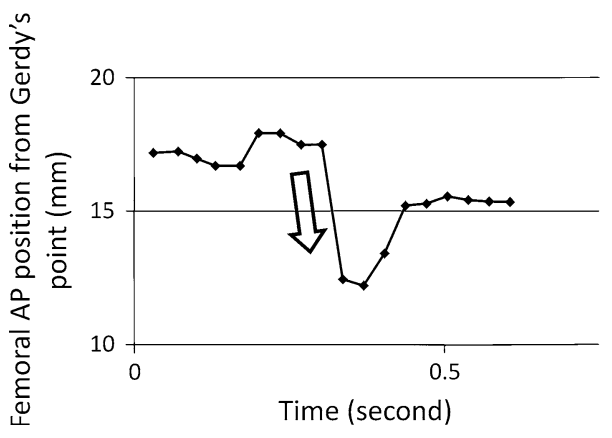

Fig. 5 Graph showing the anterior-posterior (AP) translation of the distal femur ( $y$ axis) during the pivot shift test over time ( $x$ axis). Sudden anterior translation $(5.7 \mathrm{~mm})$ of the distal femur occurs within $0.17 \mathrm{~s}$ (arrow) during the reduction phase of the manual pivot shift test

which is defined as a line connecting the centroids of Gerdy's tubercle $(\mathrm{G})$ and fibular head markers $(\mathrm{F})$, and a perpendicular line from the centroid of the lateral epicondyle marker (L) to the tibial horizontal line is calculated (Fig. 4). The ratio of the perpendicular offset length of the lateral epicondyle point from the Gerdy's tubercle point (distance "b" in Fig. 4) to the length of the tibial horizontal line (distance "a" in Fig. 4) is calculated from the $X Y$ plot data. The femoral AP position from the Gerdy's point can be calculated by multiplying the ratio by the distance of the two tibial points (from preliminary measurement).

Accuracy of the image analysis measurement

Preliminary validation of lateral compartment translation measured by our image analysis system was done using a cadaveric knee specimen in comparison with the actual bony movement that was measured by an electromagnetic tracking system $[8,14]$. In the ACL dissected knee specimen, lateral compartment translation during the pivot shift test was $3.0 \pm 0.8 \mathrm{~mm}$ for three repeated tests, whereas actual bony movement, i.e., posterior tibial translation, was $22.8 \pm 0.4 \mathrm{~mm}$. Values were smaller than the actual bony movement, but lateral compartment translation were consistently observed.

\section{Results}

Five ACL deficient knees were tested. The pivot shift test was performed by a single surgeon and evaluated as grade 1 positive. Image analysis was conducted by an individual who was blinded to the clinical grade for the pivot shift test.

The femoral AP position from the Gerdy's point suddenly decreased by $3.7 \pm 2.1 \mathrm{~mm}(\max 6.4 \mathrm{~mm} / \mathrm{min}$ $1.5 \mathrm{~mm}$ ) in the ACL deficient knees during the reduction 
phase of the pivot shift test, while the contralateral intact knees did not demonstrate such anteroposterior shift (Fig. 5). The anterior translation of the distal femur relative to the tibia occurred within $0.2 \pm 0.1 \mathrm{~s}$.

\section{Discussion}

The most important finding of the present study was that the lateral compartment translation during the pivot shift test in the ACL deficient knee was consistently observed and quantitatively measured by the newly developed image analysis technique. This study described the use of a standard digital camera system and free-of-charge image processing software to quantify the sudden anterior translation of the lateral compartment during the reduction phase of the lateral pivot shift test. As Galway et al. [5] described the lateral pivot shift phenomenon in their original report as "forward subluxation of the lateral tibial plateau on the femoral condyle and its spontaneous reduction" [5], the a-p translation in the lateral compartment is reflected in clinical grading of the pivot shift test [1]. Tracking of skin motion in this study was possible with separate markers on distal femur and proximal tibia. The different directional skin motion of bony landmarks on distal femur and proximal tibia could be detected by dynamic video motion image analysis.

There is a lack of quantitative evaluation systems for the pivot shift test, which are available for use in daily clinical practice mainly because of cost, operating space, and complexity. Navigation systems are the most commonly used device for experimental trials of kinematics measurements during the pivot shift test $[1,2,9,16]$. However, this system requires surgical intervention for fixing the tracking markers rigidly to the bones and is associated with high costs. Electromagnetic systems can be an economical alternative for accurate knee kinematics measurement during the pivot shift test $[4,7]$. Noninvasive measurement of the pivot shift is possible using simple straps [7], but their clinical use is limited by skin motion artifacts and metal interference that is extremely common in an operating or examining room environment. Although these systems have a great advantage in their capability to comprehensively examine knee kinematics during pivot shift test, the mere detection of the pivot shift movement may not require such complex systems. Based on the report that the acceleration of the pivot shift movement is related to clinical grading [7], the accelerometer has been increasingly utilized for simplified evaluation of the pivot shift test $[10,11]$.

However, these systems are exclusively for experimental use, and, even if they become available in the future, it is still unknown whether they can be economical. In contrast, the image analysis technique introduced in this report demands only a standard digital camera, free-of-charge image analysis software provided by a public institution (National Institute of Health, USA), and a few stationery stickers, which can be easily obtained.

One of the inevitable limitations of this system is the manual performance of the pivot shift test, similar to previous experimental studies for quantitative measurement of the pivot shift test $[1,2,4,7,9-11,15]$. Although an examiner might involuntarily reduce the magnitude of the pivot shift in ACL deficient knee during the manual procedure, it is difficult to elicit the pivot shift in intact knees. Therefore, as long as the examiner makes an effort to maximize the pivot shift based on his/her professional skill, the resultant knee motion should be consistent and larger when compared to external loading, such as described with the mechanized pivot shift [12]. The quantitative measurement of the pivot shift test from a "best effort" clinical examination can be used for further analysis.

Although preliminary validation study was conducted with a single specimen, the accuracy of the digital analysis measurement needs to be fully validated using larger sample size especially for the disparity between skin movement and actual translation of the bones comprising the lateral compartment, which is problematic in patients with a larger body habitus. The skin movement detected by this study is relatively small compared to the actual lateral compartment translation that is described as greater than $6 \mathrm{~mm}$ in the ACL deficient knees according to Bedi et al. [1]. This small magnitude of the skin movement might affect the sensitivity of this measurement system. Also, there was little skin movement in the short distance between Gerdy's tubercle and the fibula head in this study. Further investigation is warranted to compare the skin surface shift detected by this image analysis to bony kinematics.

Objective evaluation of the pivot shift test has been greatly needed for clinical and research needs to quantify rotational laxity in the ACL injured and reconstructed knees and could be achieved using this image analysis technique with a minimal effort and investment.

\section{Conclusion}

The sudden shift of the lateral compartment of the knee joint was successfully detected by this newly developed video-based image analysis measurement method. This image analysis technique facilitates a simple, reliable, and affordable measurement to evaluate the lateral pivot shift test. 
Open Access This article is distributed under the terms of the Creative Commons Attribution Noncommercial License which permits any noncommercial use, distribution, and reproduction in any medium, provided the original author(s) and source are credited.

\section{References}

1. Bedi A, Musahl V, Lane C, Citak M, Warren RF, Pearle AD (2010) Lateral compartment translation predicts the grade of pivot shift: a cadaveric and clinical analysis. Knee Surg Sports Traumatol Arthrosc 18(9):1269-1276

2. Bignozzi S, Zaffagnini S, Lopomo N, Fu FH, Irrgang JJ, Marcacci M (2010) Clinical relevance of static and dynamic tests after anatomical double-bundle ACL reconstruction. Knee Surg Sports Traumatol Arthrosc 18(1):37-42

3. Bull AMJ, Amis AA (1998) The pivot-shift phenomenon: a clinical and biomechanical perspective. Knee 5:141-158

4. Bull AM, Earnshaw PH, Smith A, Katchburian MV, Hassan AN, Amis AA (2002) Intraoperative measurement of knee kinematics in reconstruction of the anterior cruciate ligament. J Bone Joint Surg Br 84(7):1075-1081

5. Galway HR, Beaupre A, MacIntosh DL (1972) Pivot shift: a clinical sign of symptomatic anterior cruciate deficiency. J Bone Joint Surg Br 54-B:763-764

6. Galway HR, MacIntosh DL (1980) The lateral pivot shift: a symptom and sign of anterior cruciate ligament insufficiency. Clin Orthop Relat Res 147:45-50

7. Hoshino Y, Kuroda R, Nagamune K, Yagi M, Mizuno K, Yamaguchi M, Muratsu H, Yoshiya S, Kurosaka M (2007) In vivo measurement of the pivot-shift test in the anterior cruciate ligament-deficient knee using an electromagnetic device. Am J Sports Med 35(7):1098-1104

8. Hoshino Y, Kuroda R, Nagamune K, Nishimoto K, Yagi M, Mizuno K, Yoshiya S, Kurosaka M (2007) The effect of graft tensioning in anatomic 2-bundle ACL reconstruction on knee joint kinematics. Knee Surg Sports Traumatol Arthrosc 15(5):508-514
9. Ishibashi Y, Tsuda E, Yamamoto Y, Tsukada H, Toh S (2009) Navigation evaluation of the pivot-shift phenomenon during double-bundle anterior cruciate ligament reconstruction: is the posterolateral bundle more important? Arthroscopy 25(5):488-495

10. Labbe DR, de Guise JA, Mezghani N, Godbout V, Grimard G, Baillargeon D, Lavigne P, Fernandes J, Ranger P, Hagemeister N (2011) Objective grading of the pivot shift phenomenon using a support vector machine approach. J Biomech 44(1):1-5

11. Lane CG, Warren RF, Stanford FC, Kendoff D, Pearle AD (2008) In vivo analysis of the pivot shift phenomenon during computer navigated ACL reconstruction. Knee Surg Sports Traumatol Arthrosc 16(5):487-492

12. Maeyama A, Hoshino Y, Debandi A, Kato Y, Saeki K, Asai S, Goto B, Smolinski P, Fu FH (2011) Evaluation of rotational instability in the anterior cruciate ligament deficient knee using triaxial accelerometer: a biomechanical model in porcine knees. Knee Surg Sports Traumatol Arthrosc 19(8):1233-1238

13. Musahl V, Voos J, O'Loughlin PF, Stueber V, Kendoff D, Pearle AD (2010) Mechanized pivot shift test achieves greater accuracy than manual pivot shift test. Knee Surg Sports Traumatol Arthrosc 18(9): 1208-1213

14. Nishimoto K, Kuroda R, Mizuno K, Hoshino Y, Nagamune K, Kubo S, Yagi M, Yamaguchi M, Yoshiya S, Kurosaka M (2009) Analysis of the graft bending angle at the femoral tunnel aperture in anatomic double bundle anterior cruciate ligament reconstruction: a comparison of the transtibial and the far anteromedial portal technique. Knee Surg Sports Traumatol Arthrosc 17(3):270-276

15. Noyes FR, Grood ES, Cummings JF, Wroble RR (1991) An analysis of the pivot shift phenomenon. The knee motions and subluxations induced by different examiners. Am J Sports Med 19(2):148-155

16. Pearle AD, Kendoff D, Musahl V, Warren RF (2009) The pivotshift phenomenon during computer-assisted anterior cruciate ligament reconstruction. J Bone Joint Surg Am 91(Suppl 1):115-118 